\title{
Digital in-line holography in a droplet with cavitation air bubbles
}

\author{
S. Coëtmellec \\ coetmellec@coria.fr \\ D. Pejchang \\ D. Allano \\ G. Gréhan \\ D. Lebrun
}

\section{Brunel}

\section{A. J. E. M. Janssen}

\author{
Département d'optique, UMR-6614, Av. de l'Université, 76801 Saint-Etienne du Rouvray cedex, France \\ Département d'optique, UMR-6614, Av. de l'Université, 76801 Saint-Etienne du Rouvray cedex, France \\ Département d'optique, UMR-6614, Av. de l'Université, 76801 Saint-Etienne du Rouvray cedex, France \\ Département d'optique, UMR-6614, Av. de l'Université, 76801 Saint-Etienne du Rouvray cedex, France \\ Département d'optique, UMR-6614, Av. de l'Université, 76801 Saint-Etienne du Rouvray cedex, France
}

Département d'optique, UMR-6614, Av. de l'Université, 76801 Saint-Etienne du Rouvray cedex, France

Department of Mathematics and Computer Science, Technische Universiteit Eindhoven, 5600 MB Eindhoven, The Netherlands

In this publication, the modelisation of an air bubble as inclusion in a droplet is treated from scalar theory point of view (Fresnel's theory). The elaborated model is compared with Lorenz-Mie scattering theory and with an experimental results. Circle polynomials and scaled pupil function are the background of this work to take into account the critical angle effect that arises at a transition from a higher index to a lower index medium.

[DOI: http://dx.doi.org/10.2971/jeos.2014.14056]

Keywords: Digital in-line holography, bubble inclusions, nanoparticles

\section{INTRODUCTION}

Bubbles or air bubbling has considerable importance in liquid/gas or solid/gas systems due to their impact on the environment. The knowledge of bubble dynamics is of key importance in physical, biological, and medical processes, and in industrial applications. Sometimes bubbles are used to seed a fluid flow to study the hydrodynamics around emerging systems such as the wind or hydrokinetic systems. The behavior of bubbles and the measurement of bubble sizes in a gas-liquid flow is important. To study the bubble dynamics, optical systems that can be used include high-speed photography, for example. But other well known techniques are now used to measure simultaneously the velocity and size of a bubble. Among these techniques, we can find holography [1]-[3], the phase Doppler method [4], tomography, particle image velocimetry (PIV), and, more recently, interferometric out-of-focus imaging [5]. In particular, here we treat the use of holography when the objects are air bubbles, for the following reason: when we have a volume with an object of unknown nature, opaque particles or gas bubbles, interferometric laser imaging for droplet sizing (ILIDS) allows discriminating between these two types of objects, which is not the case with holography. This has a very important consequence, in that the resulting analysis of the distribution of the sizes of the nuclei or of the numbers of bubbles in a volume, i.e., the concentration, can be false. Probably, this impossibility is essen- tially due to the chosen experimental configuration. Recently, in the case of holography, we have proposed a modelisation of a droplet in the air to calculate the hologram of such an object [6]. In that study, the aperture, i.e., the ratio between the index of the air and the index of the droplet, is no more than unity. But in the case of an air bubble in water, the optical direction cosines, linked to $n_{d}$, of a propagating waves can be larger than unity in the water medium. When the tangential wavenumber component at the water-air interface is larger than the wavenumber in air, total internal reflection occurs and the angular spectrum of the diffracted wave spectrum is limited by this effect. Instead of a spectrum extending over the full half space, the spectrum has a limited aperture with a restriction factor given by $n_{b} / n_{d}$, the ratio of the indices of the air bubble and the water environment. Consequently, it is necessary to revise that model in order to propose a method to calculate the hologram of a gas bubble. Therefore, in the first part of this publication we recall the mathematical relation of the intensity distribution in the context of digital inline holography, and then present the necessary adaptation of our model to take into account the possibility of the numerical aperture's being greater than unity, by introducing an opaque ring. A reconstruction by the fractional Fourier transformation (FRFT) is carried out to determine an important parameter: the magnification of the optical system. This coefficient must 


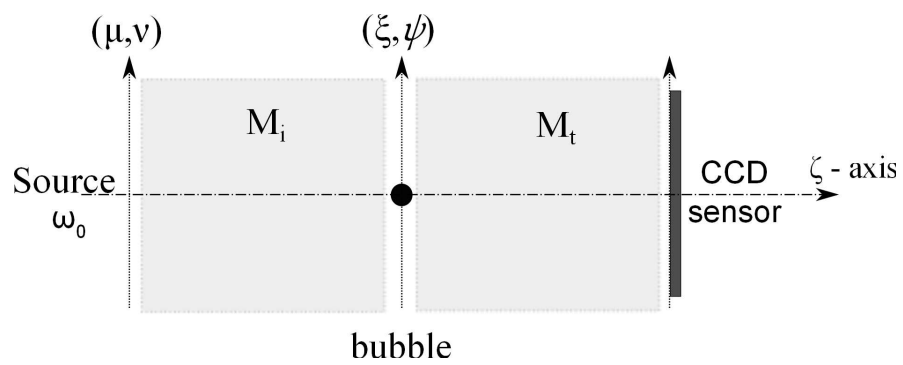

FIG. 1 Digital in-line holography setup: source $\lambda$, matrix $M_{i}$, illuminated object, matrix $M_{t}$ and $C C D$ sensor

be used to measure the real diameter of the bubble. These results have been compared with a general numerical standard of holograms of fields of particles based on the rigorous nearfield Lorenz-Mie scattering theory. In the second part of this publication, we present an experiment where nano-particles of $50 \mathrm{~nm}$ diameter are used as inclusions to produce a cavitation air bubble by means of a pulsed laser beam. Then via a reconstruction by the FRFT, a metrology of the bubble diameter is realized.

\section{PRELIMINARIES}

The optical systems for digital in-line holography are composed of two parts. Each part is delimited by a source, the object (in our case the object is a particle), and a CCD sensor. An illustration is given in Figure 1. The two parts can be fully described by two matrices, denoted $\mathbb{M}_{i}$ for the system between the laser source and the particle, and denoted $\mathbb{M}_{t}$ between the particle and the CCD sensor. The matrices $\mathbb{M}_{i}$ and $\mathbb{M}_{t}$ are defined by [7]-[9].

$$
\mathbb{M}_{i}:=\left(\begin{array}{cc}
\mathbb{A}_{i} & \mathbb{B}_{i} \\
\mathbb{C}_{i} & \mathbb{D}_{i}
\end{array}\right), \quad \mathbb{M}_{t}:=\left(\begin{array}{cc}
\mathbb{A}_{t} & \mathbb{B}_{t} \\
\mathbb{C}_{t} & \mathbb{D}_{t}
\end{array}\right)
$$

The symplecticity of the matrix $\mathbb{M}$ is defined in [10], Eq. (5) on p. 919. Here, the matrix $\mathbb{M}_{i}$ takes the form

$$
\begin{array}{ll}
\mathbb{A}_{\mathrm{i}}=\left(\begin{array}{cc}
a_{\mathrm{i}, 1} & 0 \\
0 & a_{\mathrm{i}, 2}
\end{array}\right), & \mathbb{B}_{\mathrm{i}}=\left(\begin{array}{cc}
b_{\mathrm{i}, 1} & 0 \\
0 & b_{\mathrm{i}, 2}
\end{array}\right), \\
\mathbb{C}_{\mathrm{i}}=\left(\begin{array}{cc}
c_{\mathrm{i}, 1} & 0 \\
0 & c_{\mathrm{i}, 2}
\end{array}\right), & \mathbb{D}_{\mathrm{i}}=\left(\begin{array}{cc}
d_{\mathrm{i}, 1} & 0 \\
0 & d_{\mathrm{i}, 2}
\end{array}\right)
\end{array}
$$

where $a_{\mathrm{i}, j}, b_{\mathrm{i}, j}, c_{\mathrm{i}, j}$ and $d_{\mathrm{i}, j}$ are defined by the optical components. The subscript $i$ indicates the incident part and $j$ corresponds to the transverse coordinates perpendicular to the optical axis. The subscripts 1 and 2 indicate the horizontal axis and the vertical axis, respectively. The values of the diagonal elements correspond to the cases where the system is an anamorphic or a circular optical system. In the same way, $\mathbf{M}_{t}$ takes the form

$$
\begin{array}{ll}
\mathbb{A}_{t}=\left(\begin{array}{cc}
a_{t, 1} & 0 \\
0 & a_{t, 2}
\end{array}\right), & \mathbb{B}_{t}=\left(\begin{array}{cc}
b_{t, 1} & 0 \\
0 & b_{t, 2}
\end{array}\right), \\
\mathbb{C}_{t}=\left(\begin{array}{cc}
c_{t, 1} & 0 \\
0 & c_{t, 2}
\end{array}\right), & \mathbb{D}_{t}=\left(\begin{array}{cc}
d_{t, 1} & 0 \\
0 & d_{t, 2}
\end{array}\right),
\end{array}
$$

where $a_{t, j}, b_{t, j}, c_{t, j}$ and $d_{t, j}$ are defined by the optical components from the particle plane to the CCD sensor. From
Figure 1, the propagation of the beam through can be described by two linear canonical transformations [11]. Each linear canonical transformation, denoted $\mathcal{C}$, has the same integral structure and for the first part, is given by

$$
\begin{aligned}
& \mathcal{C} {\left[G_{0}(\boldsymbol{\rho})\right](\mathbf{r})=\frac{\exp \left(i k E_{i}\right)}{i \lambda \sqrt{\operatorname{det}\left(\mathbb{B}_{i}\right)}} \int_{\mathbb{R}^{2}} G_{0}(\boldsymbol{\rho}) } \\
& \cdot \exp \left[i \frac{\pi}{\lambda}\left(\boldsymbol{\rho}^{T} \mathbb{B}_{i}^{-1} \mathbb{A}_{i} \boldsymbol{\rho}-2 \boldsymbol{\rho}^{T} \mathbb{B}_{i}^{-1} \mathbf{r}+\mathbf{r}^{T} \mathbb{D}_{i} \mathbb{B}_{i}^{-1} \mathbf{r}\right)\right] d \boldsymbol{\rho} .
\end{aligned}
$$

with $\lambda$ the wavelength and $k=2 \pi / \lambda$.

\subsection{Intensity distribution of the hologram}

The amplitude of the incident Gaussian beam, denoted $G_{i}(\mathbf{r})$, in the input plane of the inclusion, is given by [12]-[14]

$$
G_{i}(\boldsymbol{\rho})=\frac{\pi}{i \lambda} \frac{\exp \left(i k E_{i}\right)}{\sqrt{\operatorname{det}\left(\mathbb{B}_{i} \mathbb{Q}_{0}^{-1}-i \frac{\pi}{\lambda} \mathbb{A}_{i}\right)}} \cdot \exp \left[-\frac{\pi^{2}}{\lambda^{2}} \rho^{T} \mathbb{Q}_{i}^{-1} \rho\right]
$$

with

$$
\mathbb{Q}_{i}^{-1}=\mathbb{B}_{i}^{-1 T}\left(\mathbb{Q}_{0}^{-1}-i \frac{\pi}{\lambda} \mathbb{B}_{i}^{-1} A_{i}\right)^{-1} \mathbb{B}_{i}^{-1}-i \frac{\lambda}{\pi} \mathbb{D}_{i} \mathbb{B}_{i}^{-1},
$$

and the position vector in the transverse plane $\rho^{T}=\left(\begin{array}{ll}\xi & \eta\end{array}\right)$ with $|\rho|=\rho=\sqrt{\xi^{2}+\eta^{2}}$. The distance $E_{i}$ depends on the optical paths weighted by the index of the medium through which the beam has traversed. To understand the calculation process, the beam used in the holography illuminates successively the water medium of refractive index $n_{d}=1.33$, and the air bubble of refractive index $n_{b}=1$ (with $n_{d}>n_{b}$ ). The law of refraction indicates that when light is propagated from an optically denser medium into one which is optically less dense [15], a total reflexion takes place through the angle denoted by $\theta_{l}$ in Figure 2 . Otherwise, when $n_{d}<n_{b}$, total reflexion does not occur. This point has been developed in [6] in the case of a transparent particle as inclusion. In the case of [6], a general pupil function, denoted by $p(s, \theta)$, represented in the form of a Zernike series:

$$
\begin{aligned}
p(s, \theta) & =[1-A(s, \theta) \cdot \exp (i \Phi(s, \theta))] \\
& =1-\sum_{n, m} \gamma_{n}^{m} \cdot Z_{n}^{m}(s, \theta), \\
0 & \leq s \leq 1, \quad 0 \leq \theta \leq 2 \pi,
\end{aligned}
$$

where $s$ is the normalized radial coordinate and $\theta$ is the azimuthal component. The pupil function $p$ is the transmittance function between the entrance of the tangent planes and the output of the spherical object. The summation over $n$ in Eq. (7) is performed from zero to infinity and the summation over $m$ is performed from $-n$ to $n$ with $n-|m|$ even and positive. The circle polynomials, denoted here by $Z_{n}^{m}$ in Eq. (7), are given by

$$
Z_{n}^{m}(s, \theta)=R_{n}^{|m|}(s) \cdot e^{i m \theta}, \quad 0 \leq s \leq 1, \quad 0 \leq \theta \leq 2 \pi,
$$

and the Zernike coefficients $\gamma_{n}^{m}$ are obtained by using the orthogonality of the Zernike circle polynomials $Z_{n}^{m}$ :

$$
\begin{aligned}
& \gamma_{n}^{m}=\frac{n+1}{\pi} \\
& \cdot \int_{0}^{1} \int_{0}^{2 \pi} A(s, \theta) \cdot \exp [i \Phi(s, \theta)] \cdot \overline{Z_{n}^{m}(s, \theta)} s d s d \theta .
\end{aligned}
$$




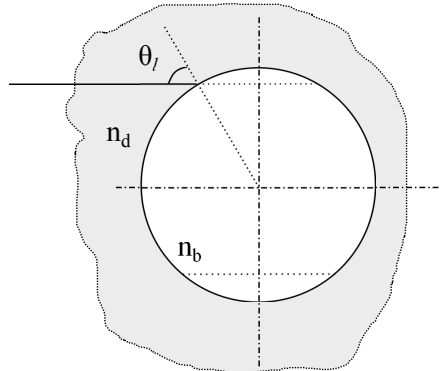

(a)

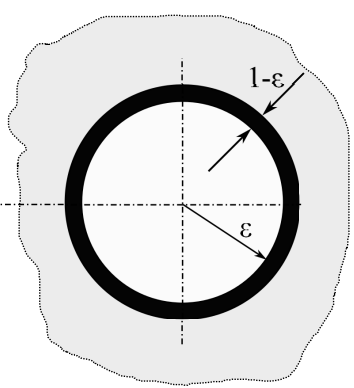

(b)
FIC. 2 Schematic representation of the numerical aperture reduced pupil function and the link to the refractive indices with $n_{d}=1.33, n_{b}=1$, (a) side view, (b) front view.

The overline represents complex conjugation. Now, to define the transmittance function of the air bubble, the total reflexion has been considered as a blind area of the ring-shaped type as illustrated in Figure 2. The normalized inner diameter, denoted $\varepsilon$, is related to the refractive indices by

$$
\varepsilon=\frac{1}{c}=\sin \left(\theta_{l}\right), \quad c=\frac{n_{d}}{n_{b}} .
$$

Consequently, the numerical aperture reduced pupil, denoted $p_{\varepsilon}$, has the following expression

$$
p_{\varepsilon}(s, \theta)=1- \begin{cases}A(s, \theta) \cdot \exp [i \Phi(s, \theta)], & 0 \leq s<\varepsilon, \\ 0 & \varepsilon<s \leq 1 .\end{cases}
$$

Now, the scaled pupil function, defined by $p_{\varepsilon}(s, \theta)$, can be represented in the form of a Zernike expansion by

$$
\begin{gathered}
p_{\varepsilon}(s, \theta)=1-\sum_{n, m} \Gamma_{n}^{m}(\varepsilon) \cdot Z_{n}^{m}(s, \theta), \\
0 \leq s \leq 1, \quad 0 \leq \theta \leq 2 \pi .
\end{gathered}
$$

Eq. (8) and the relation $[16,17]$

$$
R_{n}^{|m|}(\varepsilon s)=\sum_{n^{\prime}=|m|(2) n}\left[R_{n}^{n^{\prime}}(\varepsilon)-R_{n}^{n^{\prime}+2}(\varepsilon)\right] \cdot R_{n^{\prime}}^{|m|}(s)
$$

are combined to produce the expression of the Zernike coefficients $\Gamma_{n}^{m}(\varepsilon)$ so that

$$
\begin{gathered}
\Gamma_{n}^{m}(\varepsilon)=\frac{n+1}{\pi} \cdot \varepsilon^{2} \cdot \sum_{n^{\prime}=|m|(2) n}\left[R_{n}^{n^{\prime}}(\varepsilon)-R_{n}^{n^{\prime}+2}(\varepsilon)\right] \\
\cdot \int_{0}^{1} \int_{0}^{2 \pi} A(\varepsilon s, \theta) \cdot \exp [i \Phi(\varepsilon s, \theta)] \cdot \overline{Z_{n^{\prime}}^{m}(s, \theta)} s d s d \theta,
\end{gathered}
$$

where the integral in Eq. (14) has the same structure as the integral in Eq. (9) and if $\varepsilon=1$, then Eqs. (14) and (9) are the same. Then, the expansion coefficients $\gamma_{n}^{m}$ are used to determine the expansion coefficients $\Gamma_{n}^{m}(\varepsilon)$. The summation is over $n^{\prime}=|m|,|m|+2, \cdots, n$. This way of writing the scaled pupil function allows us to use the previous developments in [6]. Consequently, the intensity distribution $I(\sigma)$ recorded by the CCD satisfies

$$
I(\sigma)=\frac{\pi D^{2}}{4 \lambda^{2}} K \cdot\left|G(\sigma)-G_{0}^{0}(\sigma)+G_{n}^{m}(\sigma)\right|^{2}
$$

where the dimensionless variables $\sigma$ and $K$ are such that

$$
\sigma=\frac{D}{2 \lambda} \cdot \mathbb{B}_{t}^{-1} \cdot \mathbf{r}, \quad K=\left|\operatorname{det}\left(\mathbb{B}_{t}\right) \operatorname{det}\left(\mathbb{B}_{i} \mathbf{Q}_{0}^{-1}-i \frac{\pi}{\lambda} \mathbb{A}_{i}\right)\right|^{-1}
$$

with $|\mathbf{r}|=r=\sqrt{x^{2}+y^{2}}$. From Eq. (22) in [6], the three functions of the intensity in Eq. (15) are defined by,

$$
G(\sigma)=\frac{i \pi}{\sqrt{\operatorname{det}\left(\mathbb{L}_{t}\right)}} \cdot \exp \left[-i \pi^{2} \sigma^{T} \mathbb{L}_{t}^{-1} \sigma\right]
$$

with

$$
\mathbb{L}_{t}=\frac{\pi D^{2}}{4 \lambda} \mathbb{B}_{t}^{-1} \mathbb{A}_{t}+i\left(\frac{\pi D}{2 \lambda}\right)^{2} \mathbb{Q}_{i} .
$$

By noting that $\sigma=\sigma \exp (i \varphi)$, a semi-analytical expression for $G_{n}^{m}$ is then (see Appendix B)

$$
\begin{aligned}
& G_{n}^{m}(\sigma, \varphi) \\
& \quad=2 \pi \varepsilon^{2} \sum_{\mathfrak{D}} Q_{n, m, t}^{p, q}(\delta, \varepsilon) \cdot V_{t}^{m+2 q}\left(2 \pi \varepsilon \sigma, \varepsilon^{2} \chi\right) \cdot e^{i(m+2 q) \varphi},
\end{aligned}
$$

with $\mathfrak{D}$ the summation ranges for $\{n, m, p, q, t\}$ and

$$
Q_{n, m, t}^{p, q}(\delta, \varepsilon)=(-i)^{m+q} \cdot A_{n,|2 q|+2 p, t}^{m, 2 q, m+2 q} \cdot \beta_{|2 q|+2 p}^{|2 q|}\left(\varepsilon^{2} \delta\right) \cdot \Gamma_{n}^{m}(\varepsilon) .
$$

The parameter $\chi$ in Eq. (19) is the trace of the matrix $\mathbb{L}_{t}$, so that $\chi=\frac{1}{2} \cdot \operatorname{Tr}\left(\mathbb{L}_{t}\right)$. The $\delta$-parameter is linked to the ellipticity of the system, i.e., the optical components and the droplet. It is defined by $\delta=\frac{1}{2}\left(\mathbb{L}_{t(1,1)}-\mathbb{L}_{t(2,2)}\right)$ where $\mathbb{L}_{t(i, j)}$ are the diagonal elements of $\mathbb{L}_{t}$. In the particular case where the optical setup is circular, we have $\delta=0$ and $\beta_{|2 q|+2 p}^{|2 q|}(0)=1$ if $q=p=0$, and 0 otherwise. The coefficients $\beta$ can be expressed explicitly in terms of the hypergeometric functions ${ }_{2} F_{3}$ as in [29], Eqs. (A-11)-(A-13). The As in Eq. (20) are given by [19]

$$
A_{n_{1}, n_{2}, n_{3}}^{m_{1}, m_{2}, m_{3}}=\left|C_{\frac{m_{1}}{2}, \frac{m_{2}}{2}, \frac{m_{2}}{2}, \frac{n_{3}}{2}}^{\frac{m_{3}}{2}}\right|^{2},
$$

where the Cs are the Clebsch-Gordan coefficients. In the case of a symmetrical system, and in the case where the object is a spherical bubble, then $m=0$, and the function $G_{n}^{m}$ is defined by

$$
G_{n}^{0}(\sigma, \varphi)=2 \pi \varepsilon^{2} \sum_{n=0(2) \infty} \Gamma_{n}^{0}(\varepsilon) \cdot V_{n}^{0}\left(2 \pi \varepsilon \sigma, \varepsilon^{2} \chi\right),
$$

where $\mathrm{a}(2) \mathrm{b}$ denotes $a, a+2, \ldots, b$. The Zernike coefficients $\Gamma_{n}^{0}(\varepsilon)$ are defined by Eq. (14) for $m=0$. By means of Eq. (19), the $G_{0}^{0}$ function over the full disk, ie $\varepsilon=1$, takes the following expression

$$
G_{0}^{0}(\sigma, \varphi)=2 \pi V_{0}^{0}(2 \pi \sigma, \chi) .
$$

The $V$ functions have the series expression [19]

$$
\begin{aligned}
V_{n}^{m}(r, f)= & \varepsilon_{m} \exp \left(i \frac{1}{2} f\right) \cdot \sum_{k=0}^{\infty}(2 k+1) i^{k} j_{k}\left(\frac{1}{2} f\right) \\
& \cdot \sum_{h}(-1)^{\frac{m-h}{2}} \cdot A_{2 k, n, h}^{0, m, m} \frac{J_{h+1}(r)}{r} .
\end{aligned}
$$

The indices $m$ and $h$ have the same parity as $h \geq|m|$ and the $j_{k}$ are the spherical Bessel functions. The summation range over $h$ is $h=\max [|m|,|n-2 k|](1)[n+2 k]$. In Eq. (24), we have to choose $\varepsilon_{m}=-1$ for odd $m<0$ and $\varepsilon_{m}=1$ otherwise.

\subsection{Holograms of an air bubble}

In this section, we compare our developments with a rigorous near-field Lorenz-Mie scattering theory (LMT) [20]. The comparison is based on a bubble of diameter $D$ and refractive 


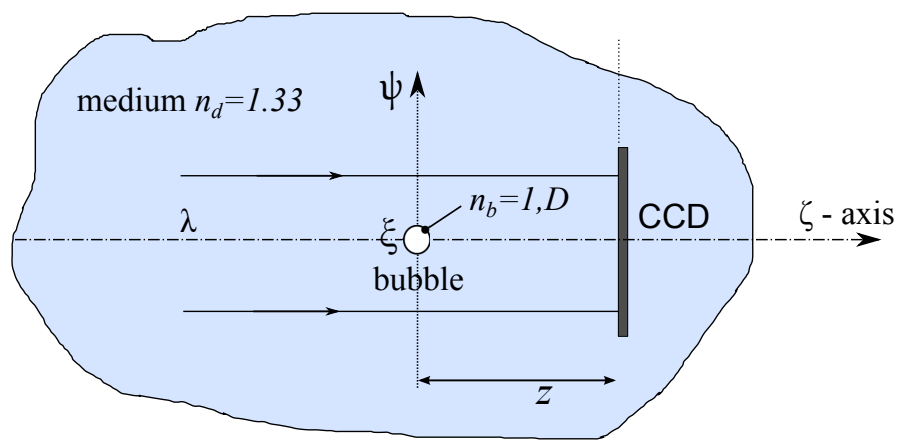

FIG. 3 Configuration under study with $n_{d}$ the refractive index of the medium and $n_{b}$ the refractive index of the bubble.

index $n_{b}$ which is equal to 1 but it is possible to choose an another refractive index, depending on the gas in the bubble. The index of the surrounding medium is denoted $n_{d}$ and equal to 1.33. In the context of the LMT, the incident beam on the particle must be a plane wave, something which modifies here only the ABCD matrices. The configuration under study is illustrated in Figure 3. In this paragraph, two cases have been considered: in the first case, the air bubble is approximated as a thin lens, ie, a quadratic phase approximation, and the second case, the air bubble is approximated as a quasi-spherical phase approximation. To elaborate the new expressions of the Zernike coefficients of the two cited cases, the Zernike coefficients in [6] for a transparent particle are used.

\subsubsection{Case of the quadratic phase approximation}

In the case of a quadratic phase approximation, we know that for a transparent particle, the pupil function over the aperture $D$ can be described by

$$
p(s, \theta)=1-\exp \left(-i \pi \kappa s^{2}\right)=1-\sum_{\substack{n=0 \\ n \text { even }}}^{\infty} \gamma_{n}^{0}(\kappa) \cdot Z_{n}^{0}(s, \theta),
$$

where $n=0,2, \ldots, \kappa=D^{2} /(4 \lambda f)$ and $f=\frac{n_{b} \cdot D}{4\left(n_{b}-n_{d}\right)}$ the effective focal length of the ball-shaped lens. The Zernike coefficients $\gamma_{n}^{0}(\kappa)$ are given by

$$
\gamma_{n}^{0}(\kappa)=(n+1) \exp \left(-i \kappa \frac{\pi}{2}\right)(-i)^{\frac{n}{2}} \cdot j_{\frac{n}{2}}\left(\kappa \frac{\pi}{2}\right) .
$$

But in the case of an air bubble considered as an inclusion in the water, due to the total reflexion, i.e., $n_{b}<n_{d}$, the definition of the scaled pupil function $p_{\varepsilon}(s, \theta)$ is obtained by combining Eqs. (26) and (14) to produce

$$
p_{\varepsilon}(s, \theta)=1-\sum_{\substack{n=0 \\ n \text { even }}}^{\infty} \Gamma_{n}^{0}(\varepsilon) \cdot Z_{n}^{0}(s, \theta)
$$

where the Zernike series $Z_{n}^{0}$ is defined on the full disc and the Zernike coefficients are such that

$$
\Gamma_{n}^{0}(\varepsilon)=\varepsilon^{2} \cdot \sum_{n^{\prime}=0(2) n}\left[R_{n}^{n^{\prime}}(\varepsilon)-R_{n}^{n^{\prime}+2}(\varepsilon)\right] \cdot \gamma_{n^{\prime}}^{0}\left(\kappa \varepsilon^{2}\right) .
$$

The Zernike coefficients $\gamma_{n^{\prime}}^{0}$ in Eq. (28) are defined by Eq. (26).

\subsubsection{Case of the quasi-spherical approximation}

Now, if the quasi-spherical approximation is employed $[6,21]$ to describe the pupil function $p$ of the air bubble, recall that in the case of [6] for a transparent particle, the pupil function $p(s, \theta)$ is defined by

$$
\begin{aligned}
p(s, \theta) & =1-\exp \left(i \pi \kappa_{n b} \sqrt{1-c^{2} \cdot s^{2}}\right) \\
& =1-\sum_{\substack{n=0 \\
n \text { even }}}^{\infty} \gamma_{n}^{0}\left(-\pi \kappa_{n b} \cdot u_{c}\right) \cdot Z_{n}^{0}(s, \theta), \\
\kappa_{n b} & =2\left(n_{b}-n_{d}\right) D / \lambda,
\end{aligned}
$$

where $c<1$ and with the Zernike coefficients

$$
\begin{aligned}
\gamma_{n}^{0}(x) & =(n+1) \cdot\left[\frac{x}{2} \cdot \mathbf{j}_{\frac{n}{2}-1} \cdot \mathbf{h}_{\frac{n}{2}}^{(2)}-\frac{x}{2 v_{c}} \mathbf{j}_{\frac{n}{2}} \cdot \mathbf{h}_{\frac{n}{2}+1}^{(2)}\right], \\
v_{c} & =\frac{1-\sqrt{1-c^{2}}}{1+\sqrt{1-c^{2}}}, \quad u_{c}=1-\sqrt{1-c^{2}} .
\end{aligned}
$$

The functions $\mathbf{j}_{n}$ are the spherical Bessel functions of the first kind and $\mathbf{h}_{n}^{(2)}$ are the spherical Hankel functions of the second kind. Each spherical Bessel function has argument $x / 2$ and each spherical Hankel function has argument $x /\left(2 v_{\mathcal{C}}\right)$. Then, the definition of the scaled pupil function $p_{\varepsilon}(s, \theta)$ for the air bubble, ie, $c>1$ or $\varepsilon<1$ is given by

$$
p_{\varepsilon}(s, \theta)=1-\sum_{\substack{n=0 \\ n \text { even }}}^{\infty} \Gamma_{n}^{0}(\varepsilon) \cdot Z_{n}^{0}(s, \theta)
$$

where the Zernike coefficients in Eq. (31) are expressed in terms of the Zernike coefficients in Eq. (30) and given by

$$
\Gamma_{n}^{0}(\varepsilon)=\varepsilon^{2} \cdot \sum_{n^{\prime}=0(2) n}\left[R_{n}^{n^{\prime}}(\varepsilon)-R_{n}^{n^{\prime}+2}(\varepsilon)\right] \cdot \gamma_{n^{\prime}}^{0}\left(-\pi \kappa_{n b}\right)
$$

with $u_{c}=v_{c}=1$ since $\varepsilon \cdot c=1$. Figure 4 illustrates the results of the quasi-spherical approximation in comparison to the LMT simulation and Figure 5 the profiles. As one can see here, the three optical signals agree quite well. The quadratic and quasi-spherical phase approximations of a bubble with the black ring thickness $\varepsilon=0.750$ give us a good approximation, close to the numerical standard with a high 2D-correlation coefficient, greater than 0.9 .

\section{RECONSTRUCTION BY FRACTIONAL FOURIER TRANSFORMATION}

The fractional Fourier transformation (FRFT) is used to reconstruct the image of an object [22]. The FRFT of order $\alpha \in \mathbb{C}$ of a function $f(r)$ is defined as [23]-[25]

$$
\begin{aligned}
& \mathcal{F}_{\alpha}[f(r)](\rho)=2 \pi C(\alpha) \cdot \exp \left[i \frac{\pi}{s^{2}} \frac{\rho^{2}}{\tan (\alpha)}\right] \\
& \cdot \int_{0}^{+\infty} f(r) \exp \left[i \frac{\pi}{s^{2}} \frac{r^{2}}{\tan (\alpha)}\right] J_{0}\left(2 \pi \frac{r \rho}{s^{2} \sin (\alpha)}\right) r d r,
\end{aligned}
$$

where $s^{2}=N \cdot \delta^{2}$, and

$$
C(\alpha)=\frac{\exp \left[-i\left(\frac{\pi}{2} \operatorname{sign}(\sin \alpha)-\alpha\right)\right]}{s^{2} \sin \alpha} .
$$

Now from the point of view of digital holography reconstruction, to reconstruct the image of the air bubble in the droplet, the fractional Fourier transformation of the intensity distribution is defined so that

$$
\mathcal{F}_{\alpha}[I(\sigma)](\rho)
$$


(a)

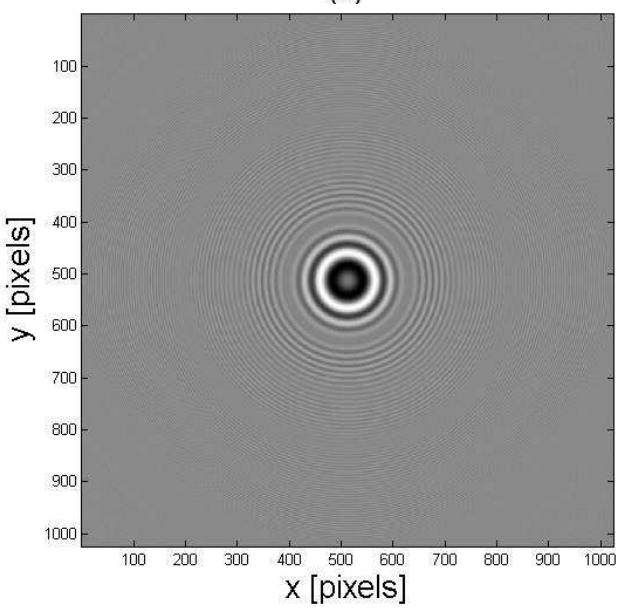

(b)

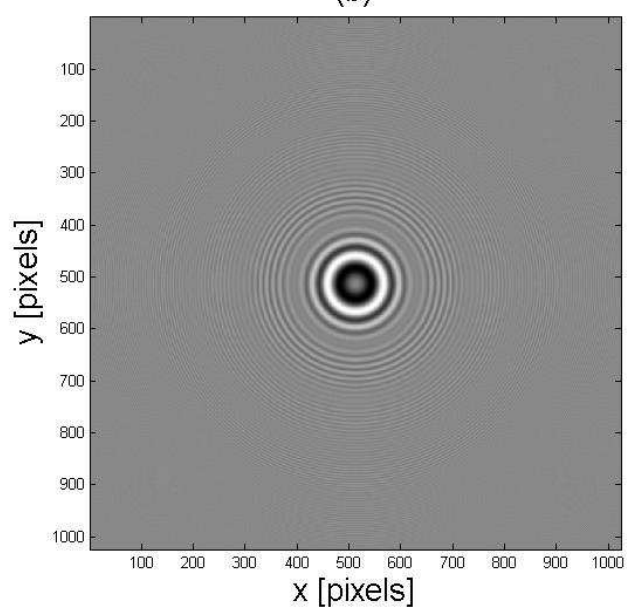

FIG. 4 Simulated intensity distribution in the plane of the CCD sensor with $\lambda=642 \mathrm{~nm}, n_{d}=1.333, n_{b}=1, D=20 \mu \mathrm{m}, z=4 \mathrm{~mm}$, (a) LMT, (b) Quasi-spherical approximation.

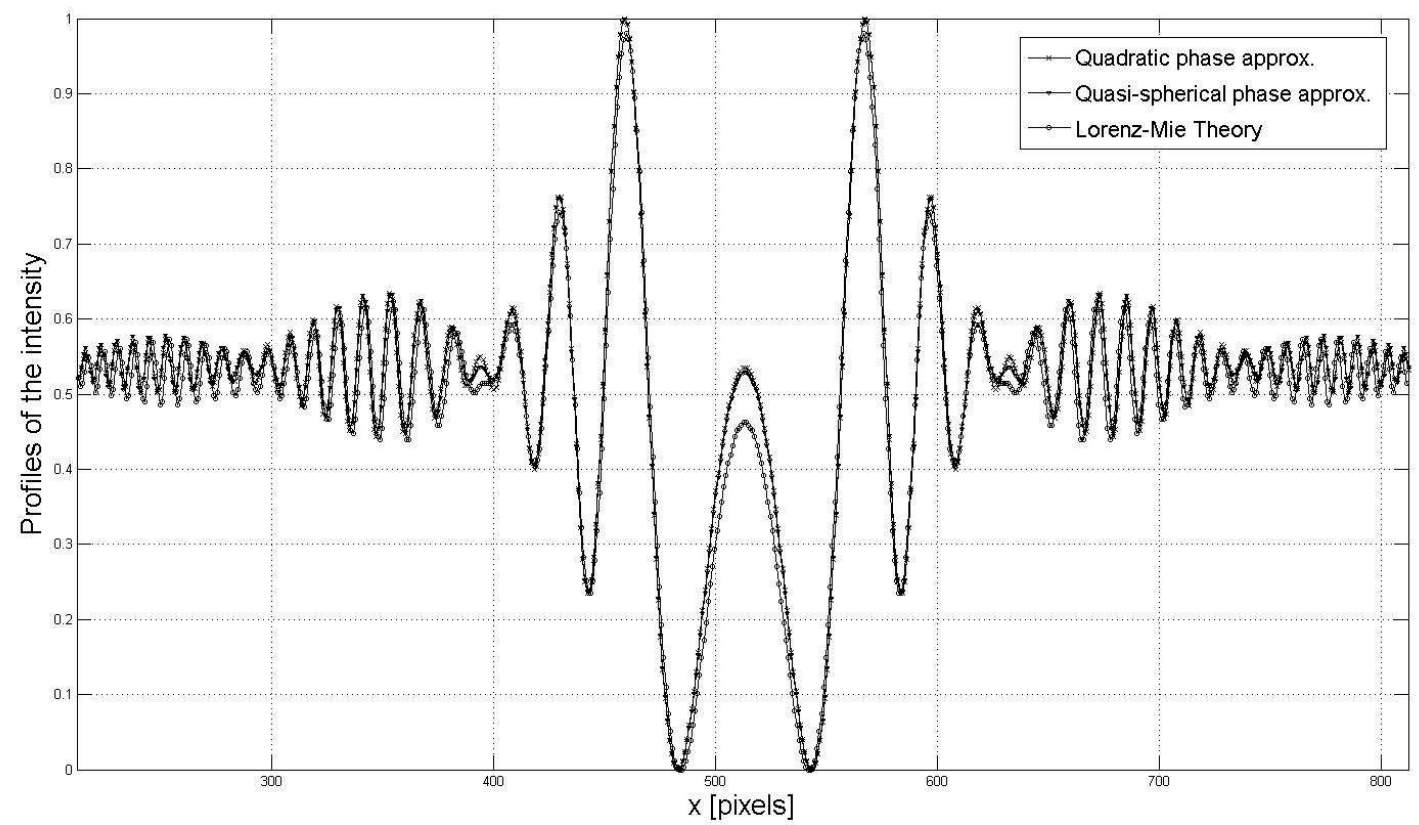

FIG. 5 Profiles of the simulated intensity distribution in the plane of the CCD sensor with $\lambda=642 \mathrm{~nm}, n_{d}=1.333, n_{b}=1, D=20 \mu \mathrm{m}, z=2 \mathrm{~mm}$ in the cases of the LMT, quadratic and quasi-spherical approximation.

From Eq. (15), the explicit expression for the intensity distribution $I$ is given by

$$
\begin{aligned}
I(\sigma)= & \frac{\pi D^{2}}{4 \lambda^{2}} K\left[|G|^{2}+\left|G_{0}^{0}\right|^{2}+\left|G_{n}^{0}\right|^{2}\right] \\
& -\frac{\pi D^{2}}{2 \lambda^{2}} K\left[\Re\left(\bar{G} G_{0}^{0}\right)+\Re\left(\bar{G} G_{n}^{0}\right)\right] \\
& -\frac{\pi D^{2}}{2 \lambda^{2}} K\left[\Re\left(\overline{G_{0}^{0}} G_{n}^{0}\right)\right]
\end{aligned}
$$

where $I$ is partially described by a real-valued chirp functions and the interferences are expressed by these real-valued chirp functions in the second term of Eq. (35). The reconstruction of the image of the air bubble is possible if we can demonstrate that the fractional Fourier transformation of the second term of Eq. (35) exhibits an aperture equal to the diameter of the air bubble object. The calculation of the fractional Fourier transform of $I(\sigma)$ to reconstruct the image of the bubble can be summarized as the calculation of the fractional Fourier transform of $\bar{G} G_{n}^{0}$ because the interference functions depend on the same chirped Bessel functions. Then, the calculation of $\bar{G} G_{n}^{0}$ gives us

$$
\begin{aligned}
& \bar{G} G_{n}^{0}=\bar{\kappa} \exp (i \chi / 2) \cdot \sum_{\mathfrak{D}} Q_{n, 0, t}^{0,0}(0, \varepsilon) \frac{J_{h+1}(2 \pi \varepsilon \sigma)}{\varepsilon \sigma} \\
& \cdot \exp \left[i \frac{\pi^{2} D^{2}}{8 \lambda^{2}} \operatorname{Tr}\left(\mathbb{B}_{t}^{-1^{T}} \overline{\mathbb{L}_{t}^{-1}} \cdot \mathbb{B}_{t}^{-1}\right) \cdot r^{2}\right]
\end{aligned}
$$

with the constant $\kappa=i \pi / \sqrt{\operatorname{det}\left(\mathbb{L}_{t}\right)}$ and the radial coordinate $\sigma=\frac{D}{4 \lambda} \operatorname{Tr}\left(\mathbb{B}_{t}^{-1}\right) r$. In the process of the reconstruction of the image of the air bubble, the quadratic phase contained in Eq. (36) must be eliminated by means of the quadratic phase of the fractional Fourier transformation. This is realized when the following conditions are satisfied:

$$
\tan \alpha_{o}= \pm \frac{8 \lambda^{2}}{\pi s^{2} D^{2}} \cdot \frac{1}{\operatorname{Tr}\left(\mathbb{B}_{t}^{-1^{T}} \frac{\mathbb{L}_{t}^{-1}}{\mathbb{B}_{t}^{-1}}\right)}
$$

Note here that the fractional order $\alpha_{o}$ is a complex fractional order and the choice of its sign \pm does not affect the reconstruction because the reconstruction is realized from real functions. Under this condition, to estimate the fractional Fourier 
(a)

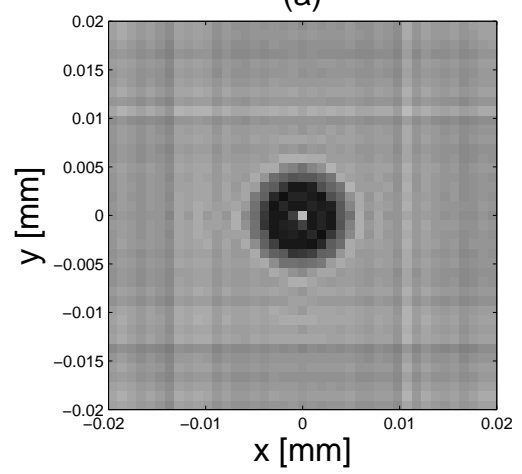

(D)

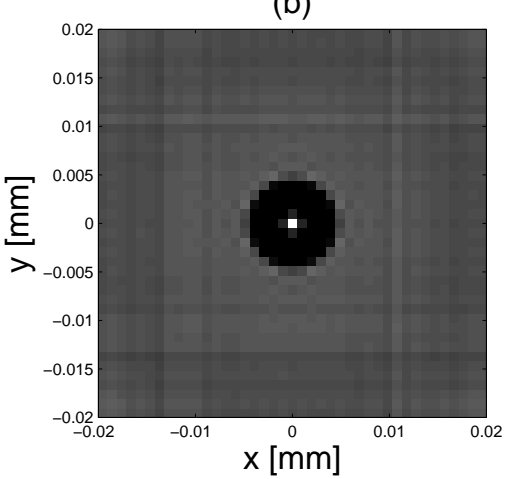

(C)

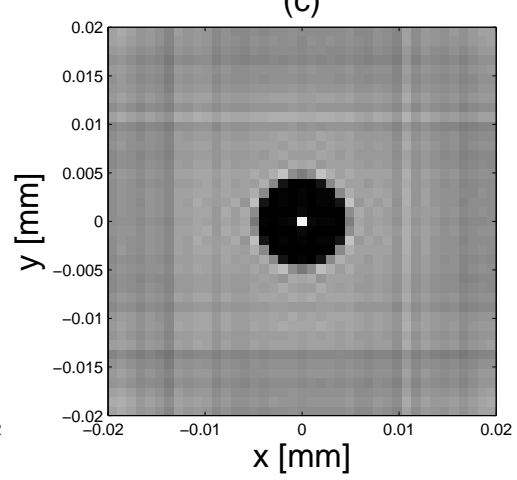

FIG. 6 Reconstruction by FRFT with $a_{x o}=a_{y o}=0.7037-i 0.73322 \cdot 10^{-7}$ in the cases of (a) the LMT, (b) the quadratic phase approximation and (c) the quasi-spherical phase approximation

transforms of Eq. (36) for the previous optimal order, we must estimate the transform

$$
\begin{aligned}
\mathcal{F}_{\alpha_{o}} & {\left[\frac{J_{h+1}(2 \pi \varepsilon \sigma)}{\sigma}\right] } \\
& \propto \int_{0}^{+\infty} J_{h+1}\left(\frac{\pi D \varepsilon}{2 \lambda} \operatorname{Tr}\left(\mathbb{B}_{t}^{-1}\right) r\right) J_{0}\left(\frac{2 \pi r \rho}{s^{2} \sin \left(\alpha_{o}\right)}\right) d r
\end{aligned}
$$

with $\sin \left(\alpha_{o}\right) \in \mathbb{C}$. Let us write $\alpha_{o}=\alpha_{o r}+i \alpha_{o i}$, where $\alpha_{o r}$ is the real part of $\alpha_{o}$ and $\alpha_{o i}$ is its imaginary part. Their expression can be deduced from Eq. (37). In practical experiments, we assume that we have the approximation $\alpha_{o r} \gg \alpha_{o i}$ with $\alpha_{o r} \in[0, \pi / 2]$ and $\alpha_{o i} \ll \pi / 2$, then $\sin \left(\alpha_{o}\right) \approx \Re\left\{\sin \left(\alpha_{o}\right)\right\}$. From [17], Eq. (7), we obtain an estimation of the integral in Eq. (38) so that

$$
\begin{aligned}
\mathcal{F}_{\alpha_{o}} & {\left[\frac{J_{h+1}(2 \pi \sigma)}{\sigma}\right] \propto \frac{2 \lambda(-1)^{\frac{h}{2}}}{\pi D \varepsilon \operatorname{Tr}\left(\mathbb{B}_{t}^{-1}\right)} \cdot R_{h}^{0}(\omega \rho), } \\
0 & <\omega \rho<1,
\end{aligned}
$$

with

$$
\omega=\frac{4 \lambda}{D \varepsilon s^{2} \Re\left(\sin \alpha_{o}\right) \operatorname{Tr}\left(\mathbb{B}_{t}^{-1}\right)} .
$$

To realize a metrology of the diameter of the bubble, the scale parameter in Eq. (40) can be used to retrieve the real diameter. This means that the parameter $\varepsilon$ must be equal to unity. The diameter $D$ of the bubble can be expressed from the estimated diameter, denoted $D_{e s t}$, in the reconstructed image, with $\varepsilon=1$, by

$$
D=G \cdot D_{\text {est }}, \quad G=\frac{2 \lambda}{s^{2} \Re\left(\sin \alpha_{o}\right) \operatorname{Tr}\left(\mathbb{B}_{t}^{-1}\right)},
$$

where $G$ is the scale factor of the optical system. Figure 6 illustrates the digital reconstructions by means of the fractional Fourier transformation from the holograms in Figures 4 and 5 of an air bubble in water and illuminated by a plane wave. The optimal fractional orders from Eqs. (37) are $a_{x o}=a_{y o}=0.7037-i 0.73322 \cdot 10^{-7}$, where the imaginary part of the optimal fractional order is very small compared to the real part.

In the case of the previous reconstruction, the estimated diameter $D_{\text {est }}$ is approximately equal to $9.15 \mu \mathrm{m}$ and with Eq. (41) of the scale factor, equal to 2.207, the theoretical diameter $D$ is equal to $20.2 \mu \mathrm{m}$, which is in line with the original diameter. Note that in the reconstructed images, we have a spot of light at the center of the bubble as in the case of the droplet in the air. This is particularly important for the subsequent study, because the spot of light inside the bubble allows us to determine that the object is really a bubble and not an opaque particle.

\subsection{Hologram of an air bubble as inclusion in a pure water droplet}

The experimental setup is represented in Figure 7. It consists of two experimental sub-setups: a digital in-line holography setup and a laser-induced nucleation setup. For the second experimental sub-setup, a single pulse of the beam of the $\mathrm{Nd}$ :YAG at $532 \mathrm{~nm}$ is focused in front of a pure water droplet $\left(\mathrm{H}_{2} \mathrm{O}\right)$ seeded with randomly positioned $\mathrm{SiO}_{2}$ nanoparticles. Their diameters are approximately equal to $50 \mathrm{~nm}$. The location of the focal point is chosen to avoid evaporation of the pure water droplet. A heating point is created in the droplet and a layer of vapor appears around the nano-particle inclusions. During cooling, the vapor grows into a cavitation air bubble. The size of the cavitation air bubble reaches a suitable diameter of several micrometers. This magnification allows us to apply the digital in-line holography technique. The mean power of the Nd:YAG is chosen to be around $0.5 \mathrm{~W}$ per pulse. The first experimental sub-setup is presented in Figure 7. The digital in-line holography is composed of two parts. The first part is delimited by the source, denoted $\omega_{0}$ and the input-plane of inclusion in the water droplet and the second is between the output-plane of the inclusion and the CCD sensor. The two parts can be fully described by the matrices $\mathbb{M}_{i}$ and $\mathbb{M}_{t}$ as previously. An illustration of an air bubble hologram created by pulses of the Nd:YAG is given in Figure 8(a) and compared to the simulated hologram in Figure 8(b). In this illustration, the diameter of the bubble inclusion is approximately $D=12.5 \mu \mathrm{m}$ and its position in the droplet is approximately $\delta=2.2 \mathrm{~mm}$. The radii of curvature of the droplet are $2.7 \mathrm{~mm}$ along the $x$-axis and $y$-axis and from the experimental optical setup, the numerical distances are $e_{0}=56 \mathrm{~mm}$, $e_{1}=242 \mathrm{~mm}, e_{2}=10.65 \mathrm{~mm}, e_{3}=5.75 \mathrm{~mm}$, and $z=39.3 \mathrm{~mm}$. In Figure 9, the simulated and experimental intensity profiles along the $x$-axis and $y$-axis are given. These profiles are obtained after normalization.

As one can see, the simulation and experiment are in very close agreement, with a $2 \mathrm{D}$ correlation coefficient equal to 


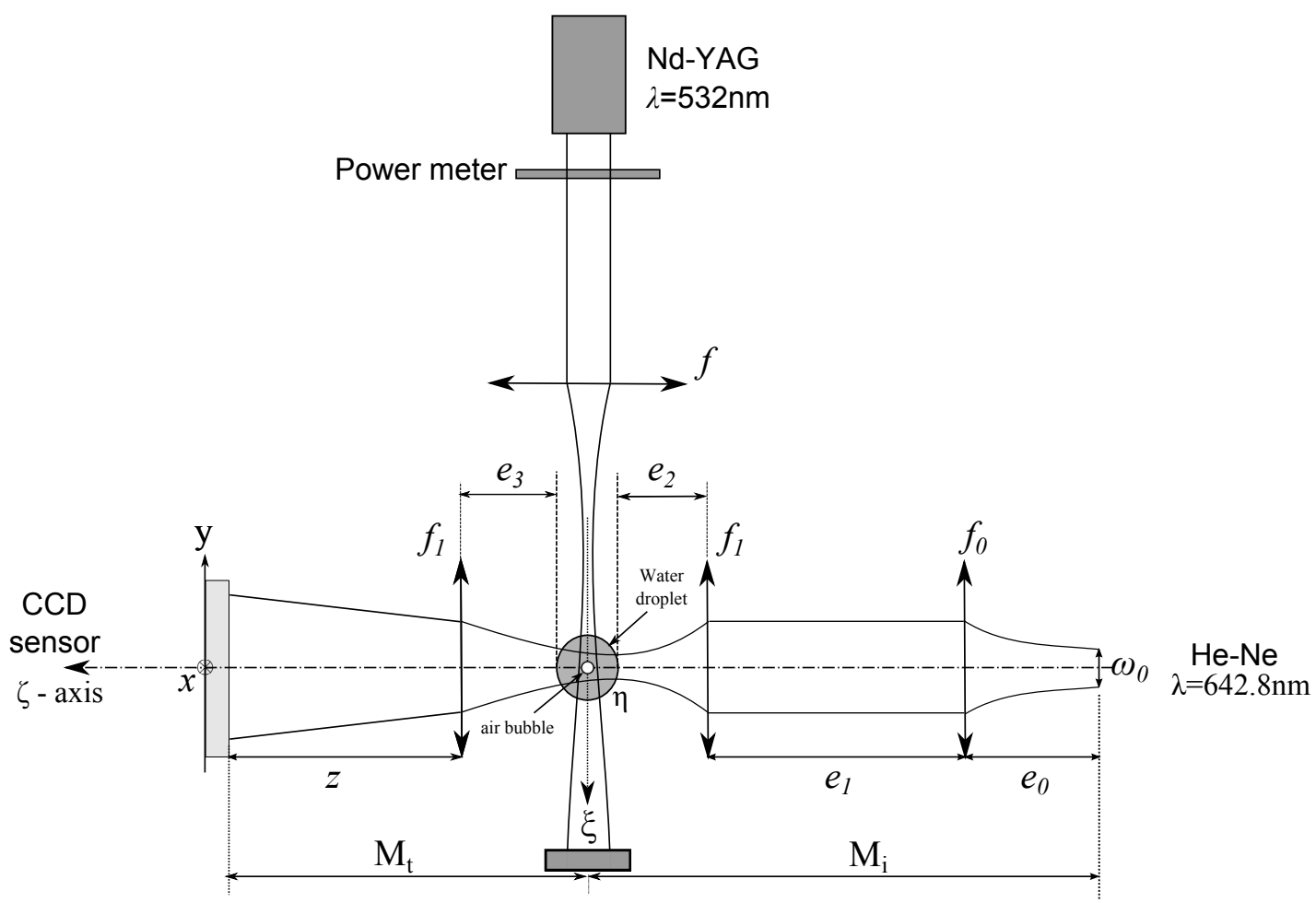

FIG. 7 Experimental setup for digital in-line holography with $\lambda=642.8 \mathrm{~nm}, \omega_{0}=2.5 \mu \mathrm{m}, f_{0}=56 \mathrm{~mm}, f_{1}=5 \mathrm{~mm}$.

(a)

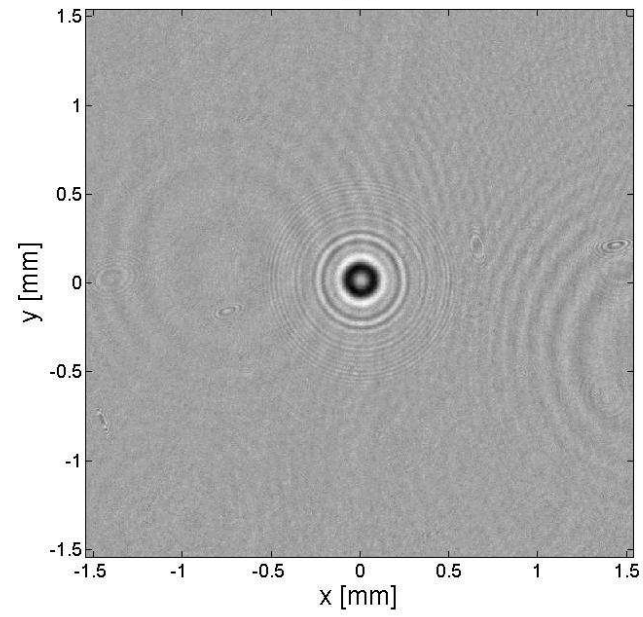

(b)

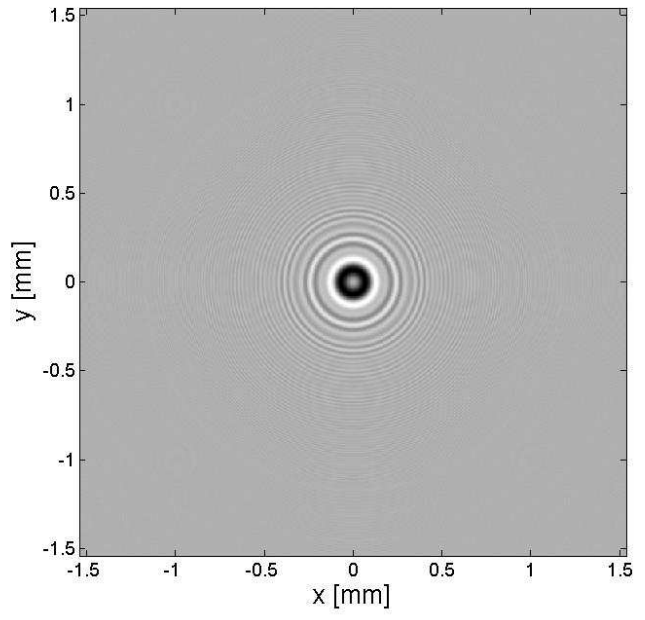

FIG. 8 Hologram of an air bubble in a droplet obtained from (a) experimental results and (b) theoretical development (quasi-shperical approximation)
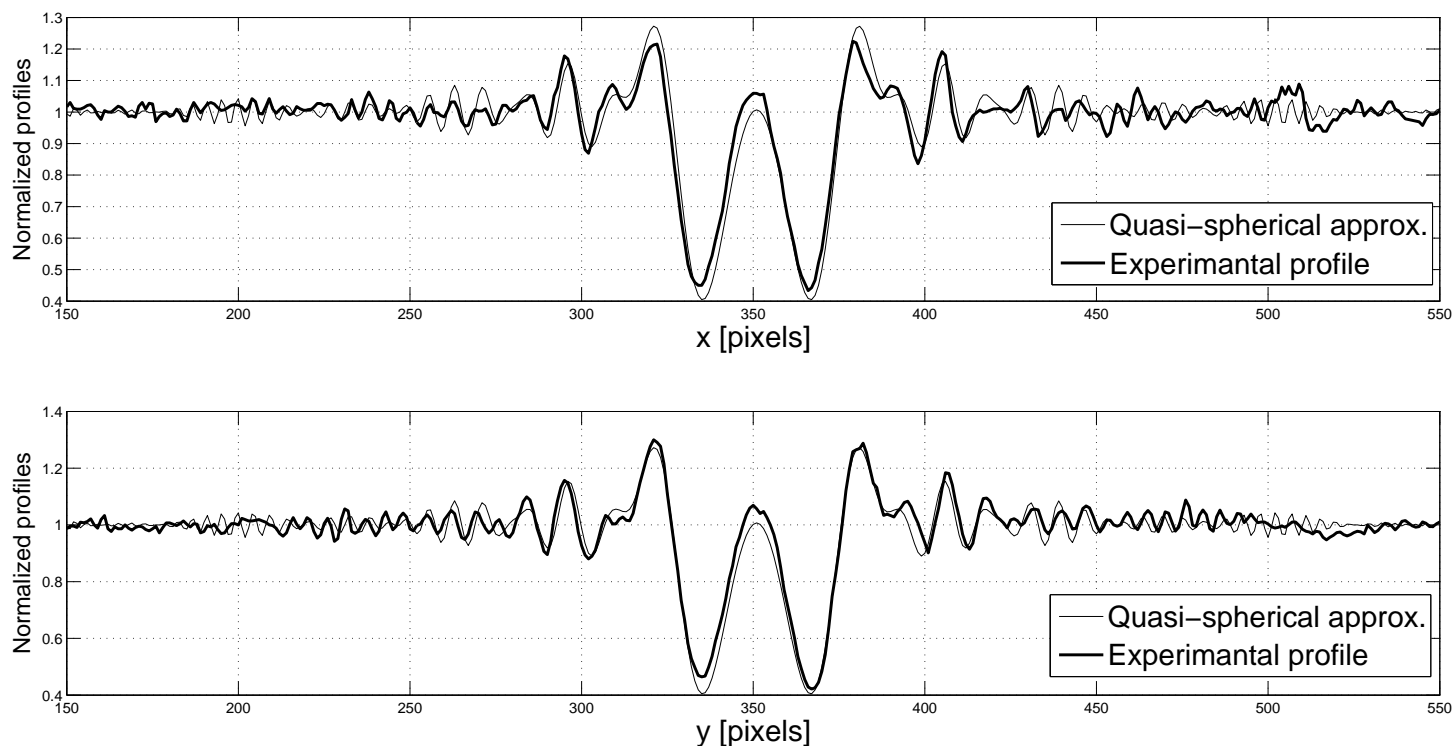

FIG. 9 Intensity profiles of the hologram of an air bubble in a droplet. 


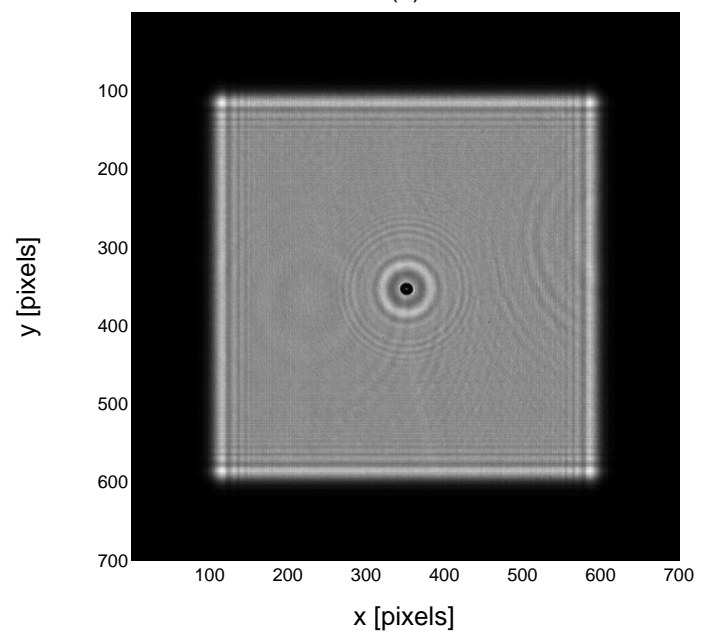

(b)

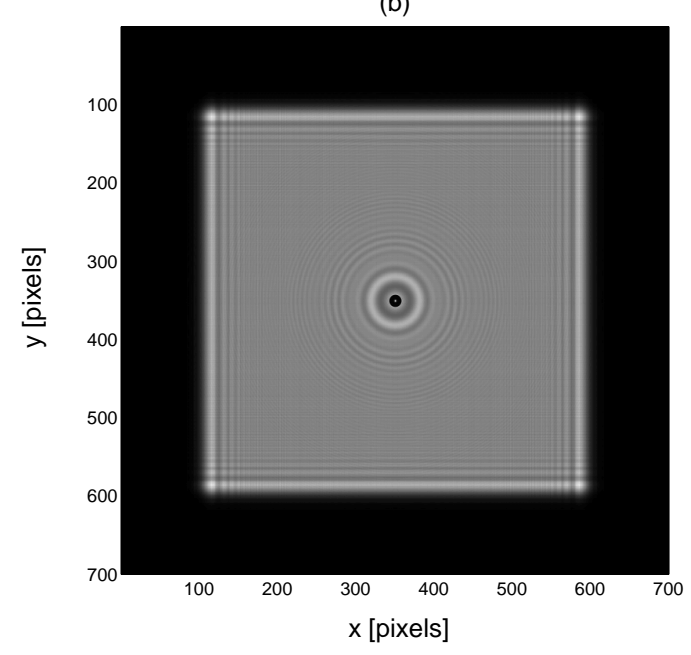

FIG. 10 Hologram of an air bubble in a droplet obtained from (a) experimental results and (b) theoretical development.

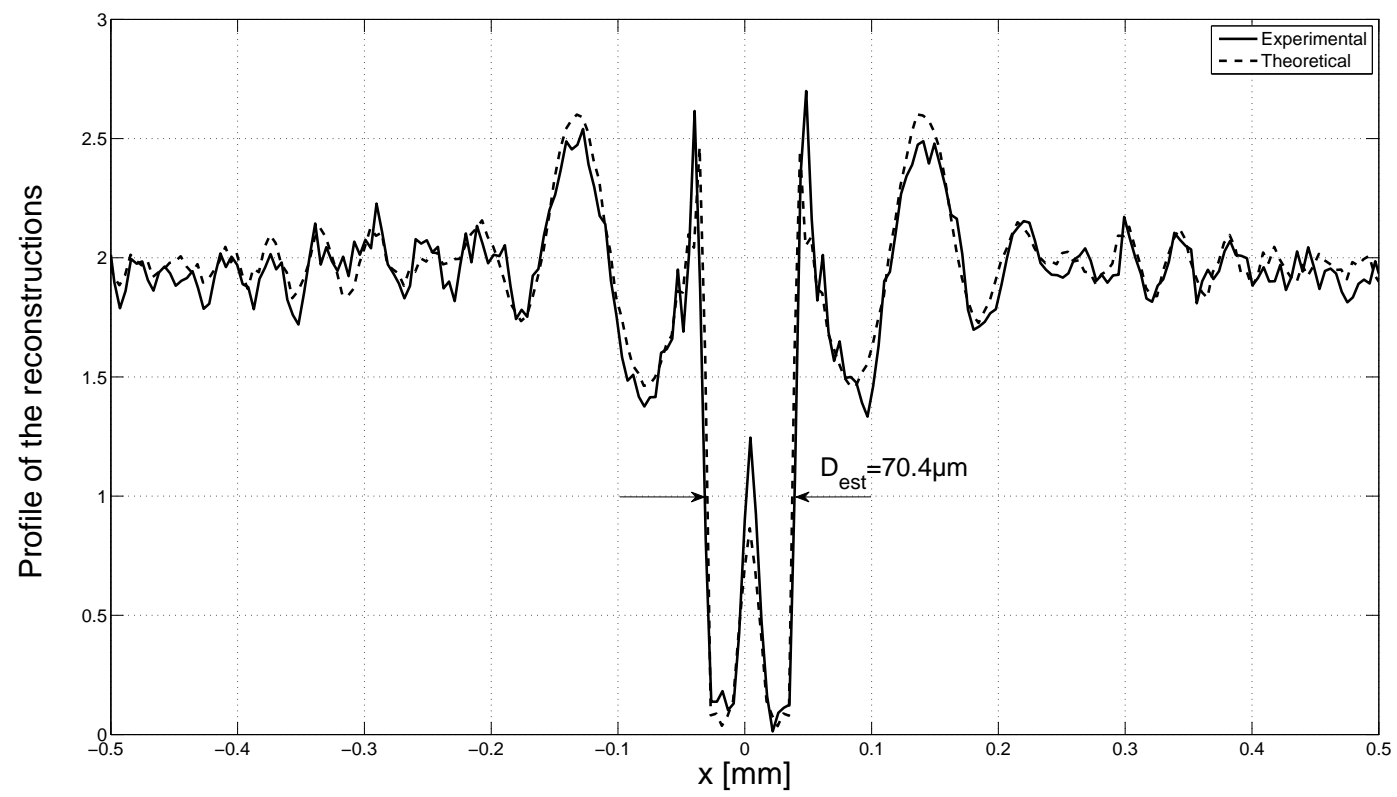

FIG. 11 Profiles of the reconstruction of the image of the air bubble: comparison between experiment and theory.

0.95 for the quasi-spherical approximation and the quadratic approximation. The quasi-spherical approximation is slightly above the quadratic approximation. This is the reason why only the quasi-spherical approximation is presented here. The reconstructed bubble inclusion images by means of the FRFT from the simulated diffraction pattern in Figure 8(b) and from the experimental diffraction pattern in Figure 8(a) are shown in Figures 10(a) and 10(b). The optimal fractional orders obtained from Eqs. (37) are $a_{x o}=a_{y o}=0.49471-\mathrm{i} 3.0459 \cdot 10^{-7}$ and with Eq. (41), the magnification factor $G$ is equal to -0.185 . To conclude, the profile of the reconstructions shown in Figure 11 to compare experiment and theory, allows us to say that the quasi-spherical approximation with a numerical aperture reduced pupil is a good approximation.

\section{CONCLUSION}

In this paper, we have proposed a mathematical model of the intensity distribution for a bubble in a liquid medium. The approximation of an opaque ring controlled by the critical angle of the total reflexion combined with the quasi-spherical ap- proximation provides a good estimation as has been demonstrated by comparison with the near-field Lorenz-Mie scattering theory. The scaled formula of the Zernike polynomials allows us to conserve the previous theoretical development presented in the case of a droplet inclusion in the air. Now, the studies are required to evaluate the experimental contexts to obtain a spot of light at the center of a bubble or of a liquid droplet.

\section{ACKNOWLEDGEMENTS}

This work was partially supported by the French National Agency under grant ANR-2011-NANO-008 "NANOMORPH." The authors acknowledge the partial financial support of the French Agence Nationale de la Recherche (ANR), through the program Investissements d'Avenir (ANR-10-LABEX-09-01). This work was partially supported by the LABEX EMC3 (Energy Materials and Clean Combustion Center) of the Universite de Rouen, within the program "3D" operated by the French National Research Agency (ANR). 


\section{A MATRICES $\mathbb{M}_{i}$ AND $\mathbb{M}_{t}$}

Here the incidence matrix is defined by means of the experimental values given in Figure 7 . Then, along the $x$-axis, the incidence matrix is defined by

$$
\begin{aligned}
\mathbb{M}_{i}= & \left(\begin{array}{cc}
1 & \frac{\delta}{n_{b}} \\
0 & 1
\end{array}\right)\left(\begin{array}{cc}
1 & 0 \\
\frac{n_{a}-n_{d}}{d / 2} & 1
\end{array}\right)\left(\begin{array}{cc}
1 & \frac{e_{2}}{n_{a}} \\
0 & 1
\end{array}\right)\left(\begin{array}{cc}
1 & 0 \\
-\frac{1}{f_{1}} & 1
\end{array}\right) \\
& \cdot\left(\begin{array}{cc}
1 & \frac{e_{1}}{n_{a}} \\
0 & 1
\end{array}\right)\left(\begin{array}{cc}
1 & 0 \\
-\frac{1}{f_{0}} & 1
\end{array}\right)\left(\begin{array}{cc}
1 & \frac{e_{0}}{n_{a}} \\
0 & 1
\end{array}\right)
\end{aligned}
$$

and along the $y$-axis, the incidence matrix is the same when the optical setup is symmetric. Now, for the transmittance matrix, we have

$$
\begin{aligned}
\mathbb{M}_{t}= & \left(\begin{array}{cc}
1 & \frac{z}{n_{a}} \\
0 & 1
\end{array}\right)\left(\begin{array}{cc}
1 & 0 \\
-\frac{1}{f_{1}} & 1
\end{array}\right)\left(\begin{array}{cc}
1 & \frac{e_{3}}{n_{a}} \\
0 & 1
\end{array}\right) \\
& \cdot\left(\begin{array}{cc}
1 & 0 \\
\frac{n_{d}-n_{a}}{-d / 2} & 1
\end{array}\right)\left(\begin{array}{cc}
1 & \frac{d_{r}-\delta}{n_{d}} \\
0 & 1
\end{array}\right),
\end{aligned}
$$

with $d_{r}$ the diameter of the droplet.

\section{B THEORETICAL DEVELOPMENT OF $G_{n}^{m}$}

The integral $G_{n}^{m}(\sigma, \varphi)$ is given by

$$
\begin{aligned}
& G_{n}^{m}(\boldsymbol{\sigma})=\int_{0}^{1} \int_{0}^{2 \pi} q_{\varepsilon}(s, \theta) \exp \left[i \frac{1}{2} s^{2}\left(\mathbb{L}_{t(1,1)}+\mathbb{L}_{t(2,2)}\right)\right] \\
& \cdot \exp \left[i \frac{1}{2} s^{2}\left(\mathbb{L}_{t(1,1)}-\mathbb{L}_{t(2,2)}\right) \cos (2 \theta)\right] \\
& \cdot \exp [-i 2 \pi r s \cos (\theta, \varphi)] s d s d \theta
\end{aligned}
$$

where the function $q_{\varepsilon}(s, \theta)=A(s, \theta) \cdot \exp [i \Phi(s, \theta)]$ with $0 \leq s<\varepsilon$ in Eq. (11). Consequently,

$$
\begin{aligned}
G_{n}^{m}(\sigma)=\int_{0}^{\varepsilon} & \int_{0}^{2 \pi} A(s, \theta) \cdot \exp [i \Phi(s, \theta)] \\
\cdot & \exp \left[i \frac{1}{2} s^{2}\left(\mathbb{L}_{t(1,1)}+\mathbb{L}_{t(2,2)}\right)\right] \\
\cdot & \exp \left[i \frac{1}{2} s^{2}\left(\mathbb{L}_{t(1,1)}-\mathbb{L}_{t(2,2)}\right) \cos (2 \theta)\right] \\
\cdot & \exp [-i 2 \pi r s \cos (\theta, \varphi)] s d s d \theta .
\end{aligned}
$$

By noting that $s^{\prime}=s / \varepsilon$, the integral $G_{n}^{m}(\sigma, \varphi)$ is

$$
\begin{aligned}
& G_{n}^{m}(\sigma)=\varepsilon^{2} \int_{0}^{1} \int_{0}^{2 \pi} A\left(\varepsilon s^{\prime}, \theta\right) \cdot \exp \left[i \Phi\left(\varepsilon s^{\prime}, \theta\right)\right] \\
& \cdot \exp \left[i \frac{1}{2} \varepsilon^{2} s^{\prime 2}\left(\mathbb{L}_{t(1,1)}+\mathbb{L}_{t(2,2)}\right)\right] \\
& \cdot \exp \left[i \frac{1}{2} \varepsilon^{2} s^{\prime 2}\left(\mathbb{L}_{t(1,1)}-\mathbb{L}_{t(2,2)}\right) \cos (2 \theta)\right] \\
& \cdot \exp \left[-i 2 \pi \varepsilon r s^{\prime} \cos (\theta, \varphi)\right] s^{\prime} d s^{\prime} d \theta .
\end{aligned}
$$

Next, from [6], the transparent particle is expressed in terms of Zernike polynomials as

$$
A(s, \theta) \cdot \exp [i \Phi(s, \theta)]=\sum_{n, m} \gamma_{n}^{m} \cdot Z_{n}^{m}(s, \theta),
$$

with the Zernike coefficients $\gamma_{n}^{m}$. But now, the air bubble can be represented in form of Zernike series as

$$
A\left(\varepsilon s^{\prime}, \theta\right) \cdot \exp \left[i \Phi\left(\varepsilon s^{\prime}, \theta\right)\right]=\sum_{n, m} \Gamma_{n}^{m}(\varepsilon) \cdot Z_{n}^{m}\left(s^{\prime}, \theta\right),
$$

where the $\Gamma_{n}^{m}(\varepsilon)$ is expressed in terms of $\gamma_{n}^{m}$ from Eq. (13). To get a semi-analytical computation method for the remaining integral in Eq. (46), the approach is as follows. Firstly, expand, using $\beta$-coefficients as before,

$$
\begin{aligned}
& \exp \left[i \frac{1}{2} \varepsilon^{2} s^{2}\left(\mathbb{L}_{t(1,1)}-\mathbb{L}_{t(2,2)}\right) \cos (2 \theta)\right] \\
& =\sum_{q=-\infty}^{+\infty} \sum_{p=0}^{\infty} i^{q} \cdot \beta_{|2 q|+2 p}^{|2 q|}\left(\varepsilon^{2} \delta\right) \cdot Z_{|2 q|+2 p}^{2 q}(s, \theta)
\end{aligned}
$$

with $\delta=\frac{1}{2}\left(\mathbb{L}_{t(1,1)}-\mathbb{L}_{t(2,2)}\right)$ and where the expansion coefficients $\beta_{|2 q|+2 p}^{|2 q|}$ are expressed explicitly in terms of the hypergeometric functions ${ }_{2} F_{3}$, cf. [29], as

$$
\begin{aligned}
& \beta_{|2 q|+2 p}^{|2 q|}(\delta)=d_{0}^{0}(-1)^{r}(2|2 q|+4 r+1)\left(\frac{1}{2} \delta\right)^{|2 q|+2 r} \\
& \cdot{ }_{2} F_{3}\left(\begin{array}{ccc}
r+\frac{1}{2} & |2 q|+r+\frac{1}{2} & \\
\frac{1}{2} & |2 q|+2 r+\frac{3}{2} & |2 q|+2 r+1
\end{array} ;-\frac{1}{4} \delta^{2}\right)
\end{aligned}
$$

in the case where $2 r-p=0$ and

$$
\begin{aligned}
& \beta_{|2 q|+2 p}^{|2 q|}(\delta)=d_{0}^{1}(-1)^{r}(2|2 q|+4 r-1)\left(\frac{1}{2} \delta\right)^{|2 q|+2 r} \\
& \cdot{ }_{2} F_{3}\left(\begin{array}{ccc}
r+\frac{1}{2} & |2 q|+r+\frac{1}{2} \\
\frac{3}{2} & |2 q|+2 r+1 & |2 q|+2 r+\frac{1}{2}
\end{array} ;-\frac{1}{4} \delta^{2}\right)
\end{aligned}
$$

in the case where $2 r-p=1$. In Eqs. (50) and (51), the coefficients $d_{0}^{0}$ and $d_{0}^{1}$ are defined as follows:

$$
\begin{aligned}
& d_{0}^{0}=\frac{(2 r) !(2|2 q|+2 r) !}{r !(|2 q|+r) !(2|2 q|+4 r+1) !^{\prime}} \\
& d_{0}^{1}=\frac{(2 r) !(2|2 q|+2 r) !}{r !(|2 q|+r) !(2|2 q|+4 r) !} .
\end{aligned}
$$

When Eq. (49) is introduced into Eq. (46), we see that there arises the product of two circle polynomials,

$$
Z_{n}^{m}(s, \theta) \cdot Z_{|2 q|+2 p}^{2 q}(s, \theta) .
$$

In [26], Eqs. (40)-(44) these products are linearized:

$$
Z_{n}^{m}(s, \theta) \cdot Z_{|2 q|+2 p}^{2 q}(s, \theta)=\sum_{t} A_{n,|2 q|+2 p, t}^{m, 2 q, m+2 q} \cdot Z_{t}^{m+2 q}(s, \theta) .
$$

The summation range over $t$ is

$$
t=\max (|m+2 q|,|m-| 2 q|-2 p|)(2)(n+|2 q|+2 p)
$$

where $\mathrm{a}(2) \mathrm{b}$ denotes $a, a+2, \ldots, b$ when $b-a$ is non-negative and even. Then, $A$ can be given in terms of Wigner or Clebsch-Gordan coefficients [27, 28]:

$$
A_{j, l, n}^{i, k, m}=\left|\begin{array}{c}
\frac{j}{2, \frac{l}{2}, \frac{n}{2}} \\
C_{\frac{i}{2}, \frac{k}{2}, \frac{m}{2}}^{2}
\end{array}\right|^{2} .
$$

The integral in Eq. (46) is now

$$
\begin{aligned}
\int_{0}^{1} \int_{0}^{2 \pi} \exp \left[i \varepsilon^{2} \chi s^{2}\right] Z_{t}^{m+2 q}(s, \theta) \\
\cdot \exp [-i 2 \pi \varepsilon \sigma s \cos (\varphi-\theta)] s d s d \theta
\end{aligned}
$$

with $\chi=\frac{1}{2} \cdot \operatorname{Tr}\left(\mathbb{L}_{t}\right)$. This latter integral can be expressed in term of $V$ functions from the extended Nijboer-Zernike theory $[29,30]$. Indeed, we have

$$
\begin{array}{r}
\int_{0}^{1} \int_{0}^{2 \pi} e^{i \varepsilon^{2} \chi s^{2}} \cdot Z_{t}^{m+2 q}(s, \theta) \cdot e^{-i 2 \pi \varepsilon \sigma s \cos (\varphi-\theta)} s d s d \theta \\
=2 \pi(i)^{m+2 q} \cdot V_{t}^{|m+2 q|}\left(2 \pi \varepsilon \sigma, \varepsilon^{2} \chi\right) \cdot e^{i(m+2 q) \varphi},
\end{array}
$$


and the power-Bessel series for $V$ is given by Eq. (24). This yields the final result of the semi-analytical formula for the integral $G_{n}^{m}$ that we were looking for:

$$
\begin{aligned}
& G_{n}^{m}\left(\sigma^{\prime}, \vartheta\right)=2 \pi \varepsilon^{2} \sum_{\mathfrak{D}}(-i)^{m+q} A_{n,|2 q|+2 p, t}^{m, 2 q, m+2 q} \\
& \cdot \beta_{|2 q|+2 p}^{|2 q|}\left(\varepsilon^{2} \delta\right) \Gamma_{n}^{m}(\varepsilon) \cdot V_{t}^{|m+2 q|}\left(2 \pi \varepsilon \sigma, \varepsilon^{2} \chi\right) \cdot e^{i(m+2 q) \varphi} .
\end{aligned}
$$

with $\mathfrak{D}$ the summation ranges for $\{n, m, p, q, t\}$.

\section{References}

[1] W. Lauterborn, and W. Hentschel, "Cavitation bubble dynamics studied by high-speed photography and holography," Ultrasonics 23, 260-268 (1985).

[2] D. Lebrun, D. Allano, L. Méès, F. Walle, F. Corbin, R. Boucheron, and D. Fréchou, "Size measurement of bubbles in a cavitation tunnel by digital in-line holography," Appl. Opt. 50, H1-H9 (2011).

[3] L. Méès, N. Grosjean, D. Chareyron, J. L. Marié, M. Seifi, and C. Fournier, "Evaporating droplet hologram simulation for digital in-line holography set-up with divergent beam," J. Opt. Soc. Am. A 30, 2021-2028 (2013).

[4] G. Gréhan, F. Onofri, T. Girasole, G. Gouesbet, F. Durst, and C. Tropea, "Measurement of bubbles by Phase Doppler technique and trajectory ambiguity," in Developments in laser techniques and applications to fluid mechanics, R. J. Adrian, D. F. G. Durão, F. Durst, M. V. Heitor, M. Maeda, and J. H. Whitelaw, eds., 290-302 (Springer-Verlag, Berlin, 1996).

[5] H. Shen, S. Saengkaew, G. Gréhan, S. Coëtmellec, and M. Brunel, "Interferometric out-of-focus imaging for the $3 \mathrm{D}$ tracking of spherical bubbles in a cylindrical channel," Opt. Commun. 320, 156-161 (2014).

[6] S. Coëtmellec, W. Wichitwong, G. Gréhan, D. Lebrun, M. Brunel, and A. J. E. M. Janssen, "Digital in-line holography assessment for general phase and opaque particle," J. Europ. Opt. Soc. Rap. Public. 9, 14021 (2014).

[7] S. A. Collins, "Lens-system diffraction integral written in terms of matrix optics," J. Opt. Soc. Am. 60, 1168-1177 (1970).

[8] T. Alieva, and M. J. Bastiaans, "Properties of the linear canonical integral transformation," J. Opt. Soc. Am. A 24, 3658-3665 (2007).

[9] R. K. Luneburg, Mathematical Theory of Optics (University of California Press, Berkeley, 1966).

[10] M. J. Bastiaans, and T. Alieva, "Signal representation on the angular Poincaré sphere, based on second-order moments," J. Opt. Soc. Am. A 27, 918-927 (2010).

[11] H. M. Ozaktas, Z. Zalevsky, and M. A. Kutay, The fractional Fourier transform: with applications in optics and signal processing (Wiley, Chichester, 2001).

[12] Y. Cai, and Q. Lin, "Propagation of elliptical Caussian beam through misaligned optical systems in spatial domain and spatialfrequency domain," Opt. Laser Technol. 34, 415-421 (2002).

[13] N. Verrier, C. Remacha, M. Brunel, D. Lebrun, and S. Coëtmellec, "Micropipe flow visualization using digital in-line holographic microscopy," Opt. Express 18, 7807-7819 (2010).
[14] N. Verrier, S. Coëtmellec, M. Brunel, D. Lebrun, and A. J. E. M. Janssen, "Digital in-line holography with an elliptical, astigmatic Gaussian beam: wide-angle reconstruction," J. Opt. Soc. Am. A 25, 1459-1466 (2008).

[15] M. Born, and E. Wolf, Principles of optics (Cambridge University Press, Cambridge, 1999).

[16] A. J. E. M. Janssen, and P. Dirksen, "Concise formula for the Zernike coefficients of scaled pupils," J. Microlith. Microfab. 5, 030501 (2006).

[17] A. J. E. M. Janssen, “New analytic results for the Zernike circle polynomials from a basic result in the Nijboer-Zernike diffraction theory," J. Europ. Opt. Soc. Rap. Public. 6, 11028 (2011).

[18] F. Nicolas, S. Coëtmellec, M. Brunel, D. Allano, D. Lebrun, and A. J. E. M. Janssen, "Application of the fractional Fourier transformation to digital holography recorded by an elliptical, astigmatic Gaussian beam," J. Opt. Soc. Am. A 22, 2569-2577 (2005).

[19] S. van Haver, and A. J. E. M. Janssen, "Advanced analytic treatment and efficient computation of the diffraction integrals in the extended Nijboer-Zernike theory," J. Eur. Opt. Soc. Rap. Public. 8, 13044 (2013).

[20] X. Wu, S. Meunier-Guttin-Cluzel, Y. Wu, S. Saengkaew, D. Lebrun, M. Brunel, L. Chen, et al., "Holography and micro-holography of particle fields: a numerical standard," Opt. Commun. 285, 3013-3020 (2012).

[21] R. M. Aarts, and A. J. E. M. Janssen, "On-axis and far-field sound radiation from resilient flat and dome-shaped radiators," J. Acoust. Soc. Am. 125, 1444-1455 (2009).

[22] S. Coëtmellec, D. Lebrun, and C. Özkul, “Characterization of diffraction patterns directly from in-line holograms with the fractional Fourier transform," Appl. Opt. 41, 312-319 (2002).

[23] V. Namias, "The fractional order Fourier transform and its application to quantum mechanics," IMA J. Appl. Math. 25, 241-265 (1980).

[24] L. Bernardo, and 0. Soares, "Optical fractional Fourier transforms with complex orders," Appl. Opt. 35, 3163-3166 (1996).

[25] P. Pellat-Finet, and E. Fogret, "Complex order fractional Fourier transforms and their use in diffraction theory. Application to optical resonators," Opt. Commun. 258, 103-113 (2006).

[26] A. J. E. M. Janssen, "Computation of Hopkins' 3-circle integrals using Zernike expansions," J. Europ. 0pt. Soc. Rap. Public. 6, 11059 (2011).

[27] M. Abramowitz, and I. Stegun, Handbook of mathematical functions (Dover Publications, New York, 1970).

[28] W. J. Tango, "The circle polynomials of Zernike and their application in optics," Appl. Phys. 13, 327-332 (1977).

[29] F. Nicolas, S. Coëtmellec, M. Brunel, D. Allano, D. Lebrun, and A. J. E. M. Janssen, "Application of the fractional Fourier transformation to digital holography recorded by an elliptical, astigmatic Gaussian beam," J. Opt. Soc. Am. A 22, 2569-2577 (2005).

[30] A. J. E. M. Janssen, "Extended Nijboer-Zernike approach for the computation of optical point-spread functions," J. Opt. Soc. Am. A 19, 849-857 (2002). 